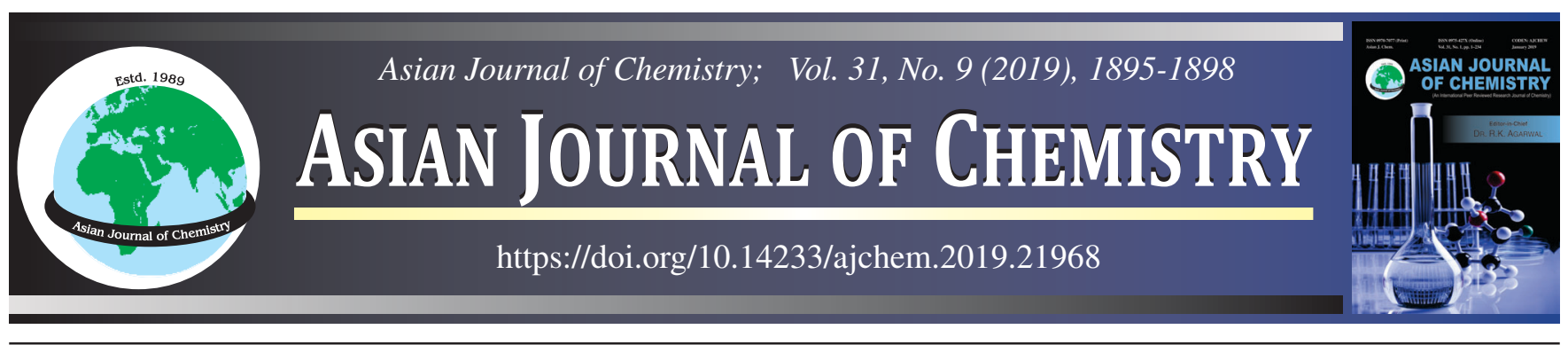

\title{
Green Synthetic Protocol for (E)-1-Aryl-3-(2-morpholinoquinolin- 3-yl)prop-2-en-1-ones and Their Antimicrobial Activity
}

\author{
Relangi Siva Subrahmanyam and Venkateswara Rao Anna*,•
}

Department of Chemistry, Koneru Lakshmaiah Education Foundation, Vaddeswaram, Guntur-522502, India

*Corresponding author: E-mail: avrchemistry@gmail.com

Received: 29 January 2019; Accepted: 22 March 2019; Published online: 31 July 2019;

We report here an easy, efficient and green synthetic protocol for the (E)-1-aryl-3-(2-morpholinoquinolin-3-yl)prop-2-en-1-ones by the Claisen-Schmidt condensation of 2-morpholinoquinoline-3-carbaldehyde and different substituted acetophenones by using 1-butyl-3methylimidazolium tetrafluoroborate $(\mathrm{Bmim}) \mathrm{BF}_{4}$. The compounds were characterized by using ${ }^{1} \mathrm{H} \mathrm{NMR},{ }^{13} \mathrm{C}$ NMR and mass spectra data and screened there in vitro antimicrobial activity against different bacterial and fungal organisms.

Keywords: Claisen-Schmidt condensation, Ionic liquid, Chalcone, Quinoline, Antimicrobial activity.

\section{INTRODUCTION}

Nitrogen-heterocycles play a predominant role in medicinal chemistry due to their pharmacological activities. The quinoline derivatives play various biological activates such as anticancer [1], anti-HIV [2], antimicrobial [3], antituberculosis [4], antiviral [5], antimalarial [6], antioxidant [7], anti-inflammatory [8], antiprotozoal [9] and some of the quinoline derivatives found in marked drugs including as chloroquine, piperaquine, pyronaridine, ciprofloxacin, lenvatinib and tipifamib. In addition, morpholine core moiety is also important in pharmacological industry due to its antimicrobial [10] and anti-inflammatory [11] activities. Furthermore, chalcone derivatives are pharmacologically active with wide variety of activates as antimicrobial [12], anticancer [13], anti-inflammatory [14], antidiabetic [15] etc. Therefore, we wish to synthesize (E)-1-aryl-3-(2morpholinoquinolin-3-yl)prop-2-en-1-ones derivatives. Generally, the synthetic approach of the chalcones involves methyl ketones and aromatic aldehydes, which is mainly catalyzed by a strong base or an acid. However, the use of strong base or acids condensation process suffers as low yields with higher reaction times and gives undesired products [16]. The chalcone also catalyzed by Lewis acids [17], Brønsted bases [18], heterogeneous catalysts [19] and metal complexes [20] but these were associated some disadvantages such as use of hazardous organic solvent system, which make the development of more greener and efficient protocol for the synthesis of these chalcones. In green chemistry concern, ionic liquids have become popular as novel and promising solvents for organic synthesis. Thus, we prompted us to synthesize (E)-1-aryl-3-(2-morpholinoquinolin-3-yl)prop-2-en-1-ones derivatives by using 1-butyl3-methylimidazolium tetrafluoroborate $(\mathrm{Bmim}) \mathrm{BF}_{4}$. The method has proved to be an easy, efficient high yields with short routine and being more environmentally-friendly.

\section{EXPERIMENTAL}

Melting points were determined in open capillary tubes and are uncorrected. The purity of the compounds was checked by TLC using precoated silica gel plates $60_{254}$ (Merck). The IR spectra were recorded on a Perkin-Elmer FT-IR-8400s, using samples in $\mathrm{KBr}$ disks. The purity of the compounds was checked by TLC using precoated silica gel plates $60_{254}$ (Merck). ${ }^{1} \mathrm{H}$ NMR and ${ }^{13} \mathrm{C}$ NMR spectra were recorded on Bruker Avance II $400 \mathrm{MHz}$ spectrometer using tetramethylsilane as an internal standard. Mass spectra were recorded on a GCMSQP 1000 EX mass spectrometer. Elemental analysis was performed on a Perkin Elmer CHN-2400 analyzer.

Synthetic procedure for (E)-1-aryl-3-(2-morpholinoquinolin-3-yl)prop-2-en-1-ones (Va-k): A mixture of 2-morpholinoquinoline-3-carbaldehyde (1) (1 mmol), aryl methyl keones (IVa-c) $(1 \mathrm{mmol})$ and $(\mathrm{Bmim}) \mathrm{BF}_{4}$ was stirred at $80{ }^{\circ} \mathrm{C}$ for 40 $50 \mathrm{~min}$. Progress of the reaction was monitored by TLC, after

This is an open access journal, and articles are distributed under the terms of the Attribution 4.0 International (CC BY 4.0) License. This license lets others distribute, remix, tweak, and build upon your work, even commercially, as long as they credit the author for the original creation. You must give appropriate credit, provide a link to the license, and indicate if changes were made. 
completion of the reaction. The reaction mixture poured into ice cold water, slowly the solid separates out, it filtered, washed with water, dried and purified by using column chromatography using $n$-hexane:ethyl acetate $(9: 1)$ to afford pure $(E)$ 1-aryl-3-(2-morpholinoquinolin-3-yl)prop-2-en-1-ones (Va-k) (Scheme-I).

(E)-3-(2-Morpholinoquinolin-3-yl)-1-phenylprop-2en-1-one (Va): Colour: yellow; yield: $90 \%$; m.p.: $127-130{ }^{\circ} \mathrm{C}$. IR $\left(\mathrm{KBr}, \mathrm{v}_{\max }, \mathrm{cm}^{-1}\right): 1652(\mathrm{C}=\mathrm{O}) ; 1586(\mathrm{C}=\mathrm{C}) ;{ }^{1} \mathrm{H}$ NMR $\left(\mathrm{CDCl}_{3}\right): 3.40-3.42$ (t, 4H, N-CH$), 3.90-3.93$ (t, 4H, O-CH$)$, 7.38-7.42 (t, 1H, ArH), 7.52-7.56 (m, 2H, ArH), 7.61-7.72 (m, 4H, ArH), 7.75-7.77 (d, 1H, ArH), 7.85-7.87 (d, 1H, ArH), 8.00-8.08 (m, 3H, ArH), 8.28 (s, $1 \mathrm{H}, \mathrm{ArH}),{ }^{13} \mathrm{C} \mathrm{NMR}\left(\mathrm{CDCl}_{3}\right)$ : 51.0, 66.9, 122.7, 123.0, 124.8, 124.9, 127.6, 127.8, 128.5, 128.6, 128.7, 130.6, 133.0, 137.2, 137.9, 141.9, 147.7, 159.6, 190.2; MS: $m / z=345(\mathrm{M}+\mathrm{H})^{+}$; Anal. Calcd. for $\mathrm{C}_{22} \mathrm{H}_{20} \mathrm{~N}_{2} \mathrm{O}_{2}$ : C, 76.72; H, 5.85; N, 8.13. Found: C, 76.78; H, 5.78; N, 8.10.

(E)-3-(2-Morpholinoquinolin-3-yl)-1-(p-tolyl)prop-2en-1-one (Vb): Colour: yellow; yield: 92 \%; m.p.: 130-133 ${ }^{\circ} \mathrm{C}$. IR $\left(\mathrm{KBr}, v_{\max }, \mathrm{cm}^{-1}\right)$ : $1651(\mathrm{C}=\mathrm{O}) ; 1590(\mathrm{C}=\mathrm{C}) ;{ }^{1} \mathrm{H}$ NMR $\left(\mathrm{CDCl}_{3}\right): 2.46$ (s, 3H, $\left.\mathrm{CH}_{3}\right), 3.40-3.42\left(\mathrm{t}, 4 \mathrm{H}, \mathrm{N}-\mathrm{CH}_{2}\right), 3.90-$ 3.93 (t, 4H, O-CH $)_{2}, 7.33-7.35$ (d, 1H, ArH), 7.38-7.42 (t, 1H, ArH), 7.63-7.77 (m, 3H, ArH), 7.85-7.87 (d, 1H, ArH), 7.978.03 (m, 3H, ArH), 8.27 (s, 1H, ArH), ${ }^{13} \mathrm{C} \mathrm{NMR}\left(\mathrm{CDCl}_{3}\right): 21.4$, 51.0, 66.9, 122.8, 123.0, 124.2, 124.7, 124.9, 125.9, 127.6, $127.8,128.7,129.4,124.8,130.5,137.2,138.4,139.0,141.4$, 144.2, 147.6, 159.6, 189.6; MS: $m / z=359(\mathrm{M}+\mathrm{H})^{+}$; Anal. Calcd. for $\mathrm{C}_{23} \mathrm{H}_{22} \mathrm{~N}_{2} \mathrm{O}_{2}$ : C, 77.07; H, 6.19; N, 7.82. Found: C, $77.12 ; \mathrm{H}, 6.14 ; \mathrm{N}, 7.85$.

(E)-1-(4-Methoxyphenyl)-3-(2-morpholinoquinolin-3yl)prop-2-en-1-one (Vc): Colour: Pale yellow, Yield: $92 \%$, m.p.: $118-121{ }^{\circ} \mathrm{C}$. IR $\left(\mathrm{KBr}, \mathrm{v}_{\max }, \mathrm{cm}^{-1}\right)$ : $1655(\mathrm{C}=\mathrm{O}) ; 1592$ $(\mathrm{C}=\mathrm{C})$; ${ }^{1} \mathrm{H}$ NMR $\left(\mathrm{CDCl}_{3}\right): 3.40-3.42\left(\mathrm{t}, 4 \mathrm{H}, \mathrm{N}-\mathrm{CH}_{2}\right), 3.91-3.93$ $\left(\mathrm{m}, 7 \mathrm{H}, \mathrm{O}-\mathrm{CH}_{2}\right), 7.00-7.02$ (d, 2H, ArH), 7.37-7.41 (t, 1H, ArH), 7.62-7.76 (m, 3H, ArH), 7.74-7.76 (d, 1H, ArH), 7.857.87 (d, 1H, ArH), 7.99-8.03 (d, 1H, ArH), 8.08-8.10 (d, 2H,
$\mathrm{ArH}), 8.26$ (s, 1H, ArH) ${ }^{13} \mathrm{C} \mathrm{NMR}\left(\mathrm{CDCl}_{3}\right): 50.9,55.5,66.9$, $113.9,122.8,122.9,124.7,124.9,127.6,127.7,130.5$, 130.7,130.8, 130.9, 137.1, 141.0, 147.6, 159.6, 163.6, 188.2; MS: $m / z=375(\mathrm{M}+\mathrm{H})^{+}$; Anal. Calcd. for $\mathrm{C}_{23} \mathrm{H}_{22} \mathrm{~N}_{2} \mathrm{O}_{3}$ : C, 73.78; H, 5.92; N, 7.48. Found: C, 73.75; H, 5.99; N, 7.53.

(E)-1-(4-Chlorophenyl)-3-(2-morpholinoquinolin-3yl)prop-2-en-1-one (Vd): Colour: Pale yellow, Yield: $89 \%$, m.p.: $136-139{ }^{\circ} \mathrm{C}$. IR (KBr, $\left.v_{\max }, \mathrm{cm}^{-1}\right)$ : $1648(\mathrm{C}=\mathrm{O}) ; 1588$ $(\mathrm{C}=\mathrm{C}) ;{ }^{1} \mathrm{H} \mathrm{NMR}\left(\mathrm{CDCl}_{3}\right): 3.40$ (s, 4H, N-CH$), 3.91$ (s, 4H, O- $\mathrm{CH}_{2}$ ), 7.38-7.43 (t, 1H, ArH), 7.50-7.52 (d, 2H, ArH), 7.647.68 (m, 2H, ArH), 7.74-7.76 (d, 1H, ArH), 7.85-7.85 (m, 1H, ArH), 8.00-8.06 (m, 3H, ArH), 8.27 (s, 1H, ArH), ${ }^{13} \mathrm{C} \mathrm{NMR}$ $\left(\mathrm{CDCl}_{3}\right)$ : 51.0, 66.9, 122.3, 122.5, 123.0, 124.8, 124.9, 127.7, 127.8, 129.0, 129.9, 130.7, 136.1, 137.3, 139.5, 142.3, 147.7, 159.6, 188.6; MS: $m / z=379(\mathrm{M}+\mathrm{H})^{+}$; Anal. Calcd. for $\mathrm{C}_{22} \mathrm{H}_{19} \mathrm{~N}_{2} \mathrm{O}_{2} \mathrm{Cl}$ : C, 69.75; H, 5.05; N, 7.39. Found: C, 69.70; $\mathrm{H}, 5.01 ; \mathrm{N}, 7.33$.

(E)-1-(4-Bromophenyl)-3-(2-morpholinoquinolin-3yl)prop-2-en-1-one (Ve): Colour: Pale yellow, Yield: $90 \%$, m.p.: $125-128{ }^{\circ} \mathrm{C}$. IR ( $\left.\mathrm{KBr}, v_{\max }, \mathrm{cm}^{-1}\right)$ : $1647(\mathrm{C}=\mathrm{O}) ; 1592$ $(\mathrm{C}=\mathrm{C}) ;{ }^{1} \mathrm{H}$ NMR $\left(\mathrm{CDCl}_{3}\right): 3.41-3.43\left(\mathrm{t}, 4 \mathrm{H}, \mathrm{N}-\mathrm{CH}_{2}\right), 3.90-3.93$ (t, 4H, O-CH $\mathrm{CH}_{2}, 3.91$ (s, 3H, O- $\left.\mathrm{CH}_{3}\right), 7.39-7.43$ (m, 1H, ArH), 7.64-7.69 (m, 4H, ArH), 7.75-7.77 (m, 1H, ArH), 7.86-7.88 (d, 1H, ArH), 7.93-7.95 (d, 2H, ArH), 8.02-8.06 (d, 1H, ArH), 8.29 (s, 1H, ArH) ${ }^{13} \mathrm{C} \mathrm{NMR}\left(\mathrm{CDCl}_{3}\right): 51.0,66.9,122.3,122.5$, 124.8, 124.9, 127.6, 127.8, 128.2, 130.0, 130.8, 132.0, 136., 137.4, 142.4, 159.5, 189.8; MS: $m / z=423(\mathrm{M}+\mathrm{H})^{+}$; Anal. Calcd. for $\mathrm{C}_{22} \mathrm{H}_{19} \mathrm{~N}_{2} \mathrm{O}_{2} \mathrm{Br}$ : C, 62.42; H, 4.52; N, 6.62. Found: C, 62.47; H, 4.56; N, 6.60.

(E)-3-(2-Morpholinoquinolin-3-yl)-1-(4-nitrophenyl)prop-2-en-1-one (Vf): Colour: Pale brown, Yield: $88 \%$, m.p.: 147-150 ${ }^{\circ} \mathrm{C}$. IR $\left(\mathrm{KBr}, \mathrm{v}_{\max }, \mathrm{cm}^{-1}\right): 1655(\mathrm{C}=\mathrm{O}), 1586(\mathrm{C}=\mathrm{C})$; ${ }^{1} \mathrm{H} \mathrm{NMR}\left(\mathrm{CDCl}_{3}\right): 3.40-3.42\left(\mathrm{t}, 4 \mathrm{H}, \mathrm{N}-\mathrm{CH}_{2}\right), 3.90-3.93$ (t, 4H, O-CH${ }_{2}$ ), 7.40-7.44 (t, 1H, ArH), 7.66-7.70 (m, 2H, ArH), 7.767.78 (d, 1H, ArH), 7.86-7.88 (d, 2H, ArH), 8.08-8.12 (d, 1H, ArH), 8.20-8.22 (d, 2H, ArH), 8.31 (s, 1H, ArH), 8.38-8.40<smiles>CC(=O)Nc1ccccc1</smiles>

II<smiles>C1COCCN1</smiles>

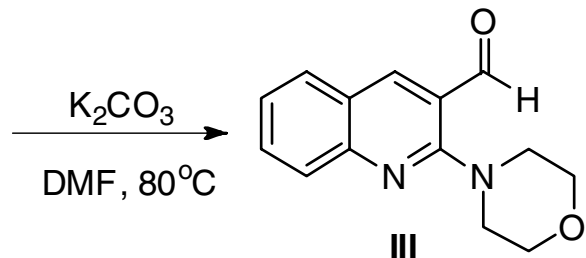

III<smiles>CC(C)C(=O)CC=Cc1cc2ccccc2nc1N1CCOCC1</smiles>

\author{
$\operatorname{Ar}=$ a) Phenyl \\ b) 4-Methyl phenyl \\ c) 4-Methoxy phenyl \\ d) 4-Chloro phenyl \\ e) 4-Bromo phenyl
}

f) 4-Nitro phenyl

g) 2-Thienyl

h) 4-Fluoro phenyl

i) 2-Naphthyl

j) 1-Naphthyl

k) 3-Methoxy phenyl

Scheme-I: Synthesis of (E)-1-aryl-3-(2-morpholinoquinolin-3-yl)prop-2-en-1-ones 
$(\mathrm{d}, 2 \mathrm{H}, \mathrm{ArH}) ;{ }^{13} \mathrm{C} \mathrm{NMR}\left(\mathrm{CDCl}_{3}\right): 51.0,66.9,123.7,124.1$, 124.7, 124.9, 127.5, 127.9, 128.5, 128.7, 129.3, 130.2, 133.2, $135.2,136.2,144.5,149.6,162.2,190.2 ; \mathrm{MS}: \mathrm{m} / z=390$ $(\mathrm{M}+\mathrm{H})^{+}$; Anal. Calcd. for $\mathrm{C}_{22} \mathrm{H}_{19} \mathrm{~N}_{3} \mathrm{O}_{4}: \mathrm{C}, 67.86 ; \mathrm{H}, 4.92 ; \mathrm{N}$, 10.79. Found: C, 67.82; H, 4.97; N, 10.82 .

(E)-3-(2-Morpholinoquinolin-3-yl)-1-(thiophen-2-yl)prop-2-en-1-one (Vg): Colour: Pale yellow, Yield: $92 \%$, m.p.: 137-140 ${ }^{\circ} \mathrm{C}$. IR $\left(\mathrm{KBr}, v_{\max }, \mathrm{cm}^{-1}\right)$ : $1644(\mathrm{C}=\mathrm{O}), 1582(\mathrm{C}=\mathrm{C})$; ${ }^{1} \mathrm{H} \mathrm{NMR}\left(\mathrm{CDCl}_{3}\right): 3.41$ (s, 4H, N-CH$)_{2}, 3.93$ (s, 4H, O-CH ${ }_{2}$ ), 3.91 (s, 3H, O- $\left.\mathrm{CH}_{3}\right), 7.23-7.26$ (t, 1H, ArH), 7.38-7.42 (t, 1H, ArH), 7.59-7.77 (m, 4H, ArH), 7.85-7.87 (d, 2H, ArH), 7.927.93 (d, 2H, ArH), 8.04-8.08 (d, 1H, O=C- $\mathrm{H}_{\alpha} \mathrm{C}=\mathrm{C}, J=16 \mathrm{~Hz}$ ), 8.26 (s, $1 \mathrm{H}, \mathrm{ArH}),{ }^{13} \mathrm{C} \mathrm{NMR}\left(\mathrm{CDCl}_{3}\right): 51.0,66.9,122.5,122.6$, 124.8, 124.9, 127.6, 127.8, 128.4, 130.6, 131.9, 134.2, 137.5, 141.2, 145.3, 147.6, 159.6, 181.7; MS: $m / z=351(\mathrm{M}+\mathrm{H})^{+}$; Anal. Calcd. for $\mathrm{C}_{20} \mathrm{H}_{18} \mathrm{~N}_{2} \mathrm{O}_{2} \mathrm{~S}$ : C, 68.55; H, 5.18; N, 7.99. Found: C, 68.50; H, 5.22; N, 7.92.

(E)-1-(4-Fluorophenyl)-3-(2-morpholinoquinolin-3yl)prop-2-en-1-one (Vh): Colour: Pale yellow, Yield: $89 \%$, m.p.: $151-154{ }^{\circ} \mathrm{C}$. IR $\left(\mathrm{KBr}, v_{\max }, \mathrm{cm}^{-1}\right)$ : $1645(\mathrm{C}=\mathrm{O}), 1587$ $(\mathrm{C}=\mathrm{C}) ;{ }^{1} \mathrm{H} \mathrm{NMR}\left(\mathrm{CDCl}_{3}\right): 3.40-3.42\left(\mathrm{t}, 4 \mathrm{H}, \mathrm{N}-\mathrm{CH}_{2}\right), 3.91-3.93$ (s, 4H, O-CH $)_{2}, 7.19-7.24(\mathrm{~m}, 2 \mathrm{H}, \mathrm{ArH}), 7.39-7.42(\mathrm{t}, 1 \mathrm{H}$, ArH), 7.64-7.70 (m, 2H, ArH), 7.75-7.77 (d, 1H, ArH), 7.857.87 (d, 1H, ArH), 8.02-8.06 (d, 1H, ArH), 8.08-8.13 (m, 2H, $\mathrm{ArH}), 8.28(\mathrm{~s}, 1 \mathrm{H}, \mathrm{ArH}),{ }^{13} \mathrm{C} \mathrm{NMR}\left(\mathrm{CDCl}_{3}\right): 51.8,66.9,121.9$, $122.9,124.3,124.6,127.5,127.8,129.0,129.9,130.5,136.3$, 137.0, 139.3, 143.1, 147.9, 160.7, 189.1; MS: $m / z=379$ $(\mathrm{M}+\mathrm{H})^{+}$; Anal. Calcd. for $\mathrm{C}_{22} \mathrm{H}_{19} \mathrm{~N}_{2} \mathrm{O}_{2} \mathrm{~F}: \mathrm{C}, 72.91 ; \mathrm{H}, 5.28 ; \mathrm{N}$, 7.73. Found: C, 72.94; H, 5.30; N, 7.77.

(E)-3-(2-Morpholinoquinolin-3-yl)-1-(naphthalen-2yl)prop-2-en-1-one (Vi): Colour: Pale yellow, Yield: $89 \%$, m.p.: $141-144{ }^{\circ} \mathrm{C}$. IR $\left(\mathrm{KBr}, v_{\max }, \mathrm{cm}^{-1}\right)$ : $1648(\mathrm{C}=\mathrm{O}), 1590$ $(\mathrm{C}=\mathrm{C}) ;{ }^{1} \mathrm{H}$ NMR $\left(\mathrm{CDCl}_{3}\right): 3.43\left(\mathrm{t}, 4 \mathrm{H}, \mathrm{N}-\mathrm{CH}_{2}\right), 3.93(\mathrm{t}, 4 \mathrm{H}, \mathrm{O}-$ $\mathrm{CH}_{2}$ ), 7.39-7.43 (t, 1H, ArH), 7.58-7.68 (m, 3H, ArH), 7.787.80 (d, 1H, ArH), 7.85-8.15 (m, 7H, ArH), 8.34 (s, 1H, ArH), 8.60 (s, $1 \mathrm{H}, \mathrm{ArH}) ;{ }^{13} \mathrm{C} \mathrm{NMR}\left(\mathrm{CDCl}_{3}\right): 51.0,66.9,122.8,122.9$, 124.3, 124.8, 125.0, 126.9, 127.7, 127.8, 127.9, 128.6, 128.7, 129.5, 130.0, 130.6, 132.5, 135.2, 135.6, 137.2, 141.8, 147.7, 159.6, 189.8; MS: $m / z=395(\mathrm{M}+\mathrm{H})^{+} ;$Anal. Calcd. for $\mathrm{C}_{26} \mathrm{H}_{22} \mathrm{~N}_{2} \mathrm{O}_{2}$ : C, 79.16; H, 5.62; N, 7.10. Found: C, 79.11; H, 5.65; N, 7.16.

(E)-3-(2-Morpholinoquinolin-3-yl)-1-(naphthalen-1yl)prop-2-en-1-one (Vj): Colour: Pale yellow, Yield: $90 \%$, m.p.: $137-140{ }^{\circ} \mathrm{C}$. IR $\left(\mathrm{KBr}, v_{\max }, \mathrm{cm}^{-1}\right): 1652(\mathrm{C}=\mathrm{O}), 1582$ $(\mathrm{C}=\mathrm{C}) ;{ }^{1} \mathrm{H}$ NMR $\left(\mathrm{CDCl}_{3}\right): 3.42$ (s, 4H, N-CH$), 3.90$ (s, 4H, O-CH 2 ), 7.20-7.22 (d, 1H, ArH), 7.37-7.45 (m, 3H, ArH), 7.58$7.73(\mathrm{~m}, 4 \mathrm{H}, \mathrm{ArH}), 7.83-7.85(\mathrm{~m}, 2 \mathrm{H}, \mathrm{ArH}), 7.94-7.96(\mathrm{~d}, 1 \mathrm{H}$, ArH), 8.06-8.12 (m, 2H, ArH), 8.23 (s, 1H, ArH); ${ }^{13} \mathrm{C}$ NMR $\left(\mathrm{CDCl}_{3}\right): 51.0,66.9,120.8,123.0,123.8,124.3,124.5,124.6$, 125.5, 125.9, 127.4, 127.5, 127.8, 130.2, 130.5, 137.3, 137.4, 142.8, 147.9, 160.7, 193.1; MS: $m / z=395(\mathrm{M}+\mathrm{H})^{+}$; Anal. Calcd. for $\mathrm{C}_{26} \mathrm{H}_{22} \mathrm{~N}_{2} \mathrm{O}_{2}$ : C, 79.16; H, 5.62; N, 7.10. Found: C, 79.21; H, 5.60; N, 7.17.

(E)-1-(3-Methoxyphenyl)-3-(2-morpholinoquinolin-3yl)prop-2-en-1-one (Vk): Colour: Pale brown, Yield: $88 \%$, m.p.: $122-125{ }^{\circ} \mathrm{C}$. IR $\left(\mathrm{KBr}, v_{\max }, \mathrm{cm}^{-1}\right): 1645(\mathrm{C}=\mathrm{O}), 1592$ $(\mathrm{C}=\mathrm{C}) ;{ }^{1} \mathrm{H} \mathrm{NMR}\left(\mathrm{CDCl}_{3}\right): 3.40-3.42\left(\mathrm{t}, 4 \mathrm{H}, \mathrm{N}-\mathrm{CH}_{2}\right), 3.90-3.93$ (m, 7H, O- $\mathrm{CH}_{2}$ ), 7.16-7.18 (dd, 1H, ArH), 7.39-7.47 (m, 2H, ArH), 7.58-7.70 (m, 4H, ArH), 7.75-7.77 (d, 1H, ArH), 7.857.87 (d, 1H, ArH), 8.00-8.04 (d, 1H, ArH), 8.28 (s, 1H, ArH), ${ }^{13} \mathrm{C} \mathrm{NMR}\left(\mathrm{CDCl}_{3}\right)$ : 51.7, 55.5, 66.9, 113.8, 113.9, 122.4, 123.3, 142.2, 124.7, 127.1, 127.4, 127.7, 130.2, 130.8, 130.9, 136.8, 141.7, 147.8, 160.7, 163.5, 188.6; MS: $m / z=375(\mathrm{M}+\mathrm{H})^{+}$; Anal. Calcd. for $\mathrm{C}_{23} \mathrm{H}_{22} \mathrm{~N}_{2} \mathrm{O}_{2}: \mathrm{C}, 73.78 ; \mathrm{H}, 5.92 ; \mathrm{N}, 7.48$. Found: C, 73.80; H, 5.95; N, 7.45.

\section{RESULTS AND DISCUSSION}

The chalcones have successfully synthesized from 2-morpholinoquinoline-3-carbaldehyde (1) and aryl methyl ketones, the intermediate aldehyde was prepared by starting from acetanilide with the reaction of DMF and $\mathrm{POCl}_{3}$ reagent followed by substitution of morppholine. The Claisen-Schimdt condensation was carried out by using 1-butyl-3-methylimidazolium tetrafluoroborate $(\mathrm{Bmim}) \mathrm{BF}_{4}$ in the place of hazardous strong bases and acids. The newly synthesized chalcone were characterized by ${ }^{1} \mathrm{H}$ NMR, ${ }^{13} \mathrm{C}$ NMR and Mass spectral data analysis. In the ${ }^{1} \mathrm{H}$ NMR spectrum the compounds (Va-k) showed characteristic newly generated two doublet in the range of $\delta 7.40$ and $7.98 \mathrm{ppm}$ integrating for each one proton was assigned for $\alpha, \beta$-unsaturated carbonyl group protons. In the ${ }^{13} \mathrm{C}$ NMR spectrum, the compounds (Va-k) showed required number of carbon peaks. The LCMS spectra exhibited the $(\mathrm{M}+\mathrm{H})^{+}$peaks their $m / z$ values.

Antibacterial activity: The synthesized compounds (Vak) were evaluated for in vitro antibacterial activity against four

TABLE-1

ANTIMICROBIAL ACTIVITY OF (E)-1-ARYL-3-(2-MORPHOLINOQUINOLIN-3-YL)PROP-2-EN-1-ONES (Va-k)

\begin{tabular}{|c|c|c|c|c|c|c|}
\hline \multirow{2}{*}{ Compound } & \multicolumn{4}{|c|}{ Antibacterial } & \multicolumn{2}{|c|}{ Antifungal } \\
\hline & S. aureus & B. subtilis & P. aeruginosa & E. coli & A. niger & Sclerotiumrol fsii \\
\hline $\mathbf{V a}$ & 16.8 & 8.8 & 17.5 & 13.3 & 14.2 & 18.8 \\
\hline $\mathbf{V b}$ & 10.3 & 9.0 & 11.2 & 10.2 & 12.8 & 14.4 \\
\hline Vc & 15.9 & 12.1 & 11.6 & 16.1 & 11.9 & 13.6 \\
\hline Vd & 9.8 & 9.4 & 13.3 & 9.9 & 11.3 & 14.2 \\
\hline Ve & 20.2 & 13.5 & 16.7 & 15.3 & 16.2 & 15.5 \\
\hline $\mathbf{V f}$ & 21.6 & 12.4 & 18.8 & 13.8 & 13.7 & 11.7 \\
\hline Vg & 18.3 & 10.0 & 9.8 & 9.6 & 10.9 & 13.4 \\
\hline Vh & 13.2 & 8.6 & 9.6 & 10.0 & 11.6 & 10.8 \\
\hline $\mathbf{V i}$ & 22.1 & 14.4 & 12.0 & 15.5 & 14.0 & 12.7 \\
\hline $\mathbf{V j}$ & 16.6 & 11.0 & 13.2 & 12.6 & 10.8 & 13.0 \\
\hline $\mathbf{V k}$ & 13.6 & 9.4 & 10.4 & 11.9 & 10.6 & 14.1 \\
\hline Norfloxacin & 25.6 & 19.2 & 24.2 & 24.0 & - & - \\
\hline Ketoconazole & - & - & - & - & 18.3 & 22.1 \\
\hline
\end{tabular}


bacterial strains Gram-positive (Staphylococcus aureus and Bacillus subtilis) and two Gram-negative (Pseudomonas aeruginosa and Escherichia coli) by paper disc method and norfloxacin used as the standard drug by measuring the zone of inhibition in $\mathrm{mm}$. The compounds were screened at the concentrations of $100 \mu \mathrm{g} / \mathrm{mL}$ in DMSO. The compounds Ve, $\mathbf{V f}, \mathbf{V g}$ and $\mathbf{V i}$ were showed good antibacterial activity against all the bacterial stains and reaming compounds showed moderated to low activity (Table-1).

Antifungal and antibacterial activities: The synthesized compounds (Va-k) were evaluated for in vitro antifungal activity against two fungal strains (Sclerotiumrolfsii and Aspergillus niger) at a concentration of $500 \mathrm{mg} / \mathrm{mL}$ by disc diffusion method, zone of inhibition measured in $\mathrm{mm}$ and ketoconazole used as the standard. Careful observation of the results shows that compounds $\mathbf{V a}, \mathbf{V e}, \mathbf{V f}$ and $\mathbf{V i}$ showed better antifungal activity and the remaining compounds were showed moderate activity against both the organisms (Table-1). The synthesized compounds were sreened their in vitro antimicrobial activity, the result suggeted that the compounds $\mathbf{V e}, \mathbf{V f}$ and $\mathbf{V i}$ showed good antimicrobial activity.

\section{Conclusion}

The use of ionic liquid $\left(\left[\mathrm{Bmim}^{\mathrm{B}} \mathrm{BF}_{4}\right)\right.$ synthetic protocal for the synthesis of chalcone deivatives offer many advantages simple reaction procedure, short rection times, high yields, exclusion of toxic solvents and easy work up.

\section{CONFLICT OF INTEREST}

The authors declare that there is no conflict of interests regarding the publication of this article.

\section{REFERENCES}

1. Y.-L. Chen, H.-M. Hung, C.-M. Lu, K.-C. Li and C.-C. Tzeng, Bioorg. Med. Chem., 12, 6539 (2004); https://doi.org/10.1016/j.bmc.2004.09.025.

2. Z. Luo, C. Zeng, W. Fang, H. He and C. Wang, Chem. Res. Chin. Univ., 25, 841 (2009).

3. N.C. Desai, B.Y. Patel and B.P. Dave, Med. Chem. Res., 26, 109 (2017); https://doi.org/10.1007/s00044-016-1732-6.
4. R.S. Keri and S.A. Patil, Biomed. Pharmacother, 68, 1161 (2014); https://doi.org/10.1016/j.biopha.2014.10.007.

5. C. de la Guardia, D. Stephens, H. Dang, M. Quijada, O. Larionov and R. Lleonart, Molecules, 23, 672 (2018); https://doi.org/10.3390/molecules23030672.

6. M. Foley and L. Tilley, Pharmacol. Ther, 79, 55 (1998); https://doi.org/10.1016/S0163-7258(98)00012-6.

7. M.O. Püsküllü, B. Tekiner and S. Suzen, Mini Rev. Med. Chem., 13, 365 (2013);

https://doi.org/10.2174/138955713804999793.

8. S. Mukherjee and M. Pal, Curr. Med. Chem., 20, 4386 (2013); https://doi.org/10.2174/09298673113209990170.

9. E. García, J.C. Coa, E. Otero, M. Carda, I.D. Vélez, S.M. Robledo and W.I. Cardona, Med. Chem. Res., 27, 497 (2018); https://doi.org/10.1007/s00044-017-2076-6.

10. H. Bektas, S. Ceylan, N. Demirbas, S. Alpay-Karaoglu and B.B. Sökmen, Med. Chem. Res., 22, 3629 (2013); https://doi.org/10.1007/s00044-012-0318-1.

11. A. Ahmadi, M. Khalili, R. Hajikhani, N. Safari and B. Nahri-Niknafs, Med. Chem. Res., 21, 3532 (2012); https://doi.org/10.1007/s00044-011-9891-y.

12. D.D. Bozic, M. Milenkovic, B. Ivkovic and I. Cirkovic, Indian J. Med. Res., 140, 130 (2014).

13. C. Karthikeyan, N.S.H. Narayana Moorthy, S. Ramasamy, U. Vanam, E. Manivannan, D. Karunagaran and P. Trivedi, Recent Patents Anticancer Drug Discov., 10, 97 (2014); https://doi.org/10.2174/1574892809666140819153902.

14. H.K. Hsieh, L.T. Tsao, J.P. Wang and C.N. Lin, J. Pharm. Pharmacol., 52, 163 (2000); https://doi.org/10.1211/0022357001773814.

15. C.T. Hsieh, T.J. Hsieh, M. El-Shazly, D.W. Chuang, Y.H. Tsai, C.T. Yen, S.F. Wu, Y.C. Wu and F.R. Chang, Bioorg. Med. Chem. Lett., 22, 3912 (2012); https://doi.org/10.1016/j.bmcl.2012.04.108.

16. B.A. Hathaway, J. Chem. Educ., 64, 367 (1987); https://doi.org/10.1021/ed064p367.

17. M.A. Bigdeli, G.H. Mahdavinia, S. Jafari and H. Hazarkhani, Catal. Commun., 8, 2229 (2007); https://doi.org/10.1016/j.catcom.2007.05.010.

18. S. Bhagat, R. Sharma and A.K. Chakraborti, J. Mol. Catal. Chem., 260, 235 (2006); https://doi.org/10.1016/j.molcata.2006.07.018.

19. A. Fischer, P. Makowski, J.-O. Müller, M. Antonietti, A. Thomas and F. Goettmann, ChemSusChem, 1, 444 (2008); https://doi.org/10.1002/cssc.200800019.

20. T. Mukaiyama, K. Narasaka and H. Hokonoki, J. Am. Chem. Soc., 91, 4315 (1969); https://doi.org/10.1021/ja01043a066. 